\title{
Using Sepsis-3 criteria to predict prognosis of patients receiving continuous renal replacement therapy for community- acquired sepsis: a retrospective observational study
}

\author{
Maho Akiu ${ }^{1,2}$, Tae Yamamoto ${ }^{1,2^{*}}$, Mariko Miyazaki ${ }^{1,2}$, Kimio Watanabe $^{2}$, Emi Fujikura ${ }^{1,2}$, Masaaki Nakayama ${ }^{3}$, \\ Hiroshi Sato ${ }^{1}$ and Sadayoshi Ito ${ }^{1}$
}

\begin{abstract}
Background: The definition and guideline of sepsis and septic shock were recently updated. The aim of this study is to evaluate the ability of Third Consensus Definitions of Sepsis and Septic Shock (Sepsis-3) to predict outcomes among patients with community-acquired sepsis receiving continuous renal replacement therapy (CRRT).

Methods: We conducted a retrospective observational study between January 2013 and December 2015 in a single university hospital. From 368 patients receiving CRRT for various reasons, 64 patients who suffered from communityacquired sepsis and required CRRT were selected and evaluated using the current and previous sepsis criteria. We additionally assessed infection characteristics. The primary outcome was 28-day mortality, and the secondary outcome was in-hospital mortality.
\end{abstract}

Results: Of the 64 participants (70.3\% male, median age 66.5 years), 33 (51.6\%) administered antimicrobials before admission. The most common source of infections was pneumonia, and 27 participants (42.2\%) had positive cultures. The Sepsis-3 criteria identified 64 cases (100\%) as sepsis at the start of CRRT, while the previous criteria identified 44 cases (68.8\%). According to the Sepsis-3 criteria, the 28-day mortality of sepsis and septic shock were $31.3 \%(20 / 64)$ and $46 \%$ (17/37), and in-hospital mortality was $43.8 \%(28 / 64)$ and $62.2 \%$ (23/37), respectively. Septic shock diagnosed using the Sepsis-3 criteria predicted mortality (log-rank $P=0.0001$ ); however, using the previous criteria was not associated with mortality (log-rank $P=0.437$ ). Among variables, lactate levels $\geq 2 \mathrm{mmol} / \mathrm{L}$ and SOFA score $\geq 14$ were significantly associated with mortalities, with an optimal cutoff value for lactate of $1.8 \mathrm{mmol} / \mathrm{L}$ (AUC 0.777 , sensitivity $85.7 \%$, specificity $58.3 \%$ ). Although age $\geq 65$ years predicted in-hospital mortality, and pre-hospital antimicrobial therapy tended to be associated with 28-day mortality, we did not detect any association between outcomes and the CRRT regimen or general risk factors (e.g., acute kidney injury, serum creatinine levels, and comorbidities).

(Continued on next page)

\footnotetext{
* Correspondence: tae.yamamoto@med.tohoku.ac.jp

${ }^{1}$ Division of Nephrology, Endocrinology and Vascular Medicine, Tohoku

University Graduate School of Medicine, 1-1 Seiryomachi, Aoba-ku, Sendai,

Miyagi 980-8574, Japan

${ }^{2}$ Division of Blood Purification, Tohoku University Hospital, Sendai, Japan

Full list of author information is available at the end of the article
}

(c) The Author(s). 2018 Open Access This article is distributed under the terms of the Creative Commons Attribution 4.0 International License (http://creativecommons.org/licenses/by/4.0/), which permits unrestricted use, distribution, and reproduction in any medium, provided you give appropriate credit to the original author(s) and the source, provide a link to the Creative Commons license, and indicate if changes were made. The Creative Commons Public Domain Dedication waiver (http://creativecommons.org/publicdomain/zero/1.0/) applies to the data made available in this article, unless otherwise stated. 
(Continued from previous page)

Conclusions: Our data suggests that the Sepsis-3 criteria predicted survival more accurately than the previous criteria among patients with community-acquired sepsis receiving CRRT. This is based on lactate levels and SOFA scores being strongly associated with mortality.

Keywords: Mortality, Septic shock, Lactate, SOFA

\section{Background}

Sepsis and septic shock are the most common contributing factors to acute kidney injury (AKI) in critically ill patients and account for approximately $50 \%$ of AKI cases in intensive care units (ICU) [1]. Septic AKI has a higher mortality rate than non-septic AKI [2-5]. Continuous renal replacement therapy (CRRT) is used to address sepsis-related renal failure and improve outcomes by stabilizing circulation and removing inflammatory mediators [6]. It has been reported that early diagnosis and treatment using appropriate antimicrobials can reduce mortality from sepsis [7-9]. Although early initiation of renal replacement therapy may contribute to reduced mortality [10-12], there is limited evidence about the usefulness of CRRT in sepsis [13-15]. Recent reports have clarified clinical features associated with mortality in patients with sepsis-related AKI receiving CRRT [16]. However, most of these studies included patients with nosocomial sepsis, such as postoperative cases, and there are few reports about clinical features and prognosis of patients who required CRRT for community-acquired sepsis.

Definitions of sepsis and septic shock have evolved since the early 1990s. Sepsis-1 [17] and Sepsis-2 [18] defined sepsis as a systemic inflammatory response caused by infection, and the systemic inflammatory response syndrome (SIRS) criteria were used to identify cases of sepsis. However, in 2016, the Third Consensus Definitions of Sepsis and Septic Shock (Sepsis-3) provided a more precise approach to diagnosing sepsis [19]. Sepsis3 defines sepsis as a life-threatening organ dysfunction caused by a dysregulated host response to infection and recommends using the Sequential Organ Failure Assessment (SOFA) score to monitor patients in the ICU for organ dysfunction and sepsis. A simpler quick SOFA (qSOFA) score has also been developed for use in nonICU patients with infection, and lactate levels have become indispensable for diagnosing septic shock.

Some studies have compared the utility of the Sepsis-3 criteria to that of the previous definitions [20]. However, few studies have investigated whether Sepsis-3 is useful for evaluating patients who require CRRT. Therefore, the present study examined the characteristics of patients who required CRRT for community-acquired sepsis and assessed the ability of Sepsis- 1 and Sepsis- 3 to predict clinical outcomes.

\section{Methods}

Study design

This study was a retrospective observational study conducted at the Tohoku University Hospital, a university hospital in Miyagi Prefecture, Japan. The study was approved by the Ethics Committee of the Tohoku University Graduate School of Medicine (2016-1-008).

\section{Participant selection}

Patients attending the ICU or emergency department (ED) between January 2013 and December 2015 who had primary infections and performed CRRT were screened for inclusion in this study. During the study period, 368 patients performed CRRT for various reasons. As shown in Fig. 1, the exclusion criteria were cardiopulmonary arrest on admission, burns, traffic injuries, drug poisoning, heat stroke, organ transplantation surgery (including bone marrow transplantation), age of $<18$ years, pregnancy, chronic maintenance dialysis, previous renal transplantation, and insufficient data at admission. We also excluded 86 patients who performed CRRT for non-infectious diseases and a further 111 who had secondary infections, such as nosocomial infections that developed after a scheduled surgery or examination, and the underlying disease for hospitalization. We ultimately identified 64 patients with primary infectious diseases who started CRRT within $72 \mathrm{~h}$ of hospitalization, who became our study participants (Fig. 1).

\section{Data collection}

We used medical records for the 64 participants to collect the following information: age, sex, body mass index, underlying medical conditions, date and cause of admission, time and reason for CRRT administration, CRRT type, clinical and physiological data the time of hospitalization and the start of CRRT, origin of infection, laboratory data at the start of CRRT (including lactate levels), Acute Physiology and Chronic Health Evaluation (APACHE) II score [21], and SOFA score [22].

\section{Sepsis definitions}

We used Sepsis-3 and Sepsis-1 criteria to diagnose sepsis and septic shock and compared the results. Sepsis-1 was used as the reference because previous studies had reported that Sepsis-1 and Sepsis-2 had similar diagnostic performances, but Sepsis-1 was simpler and 


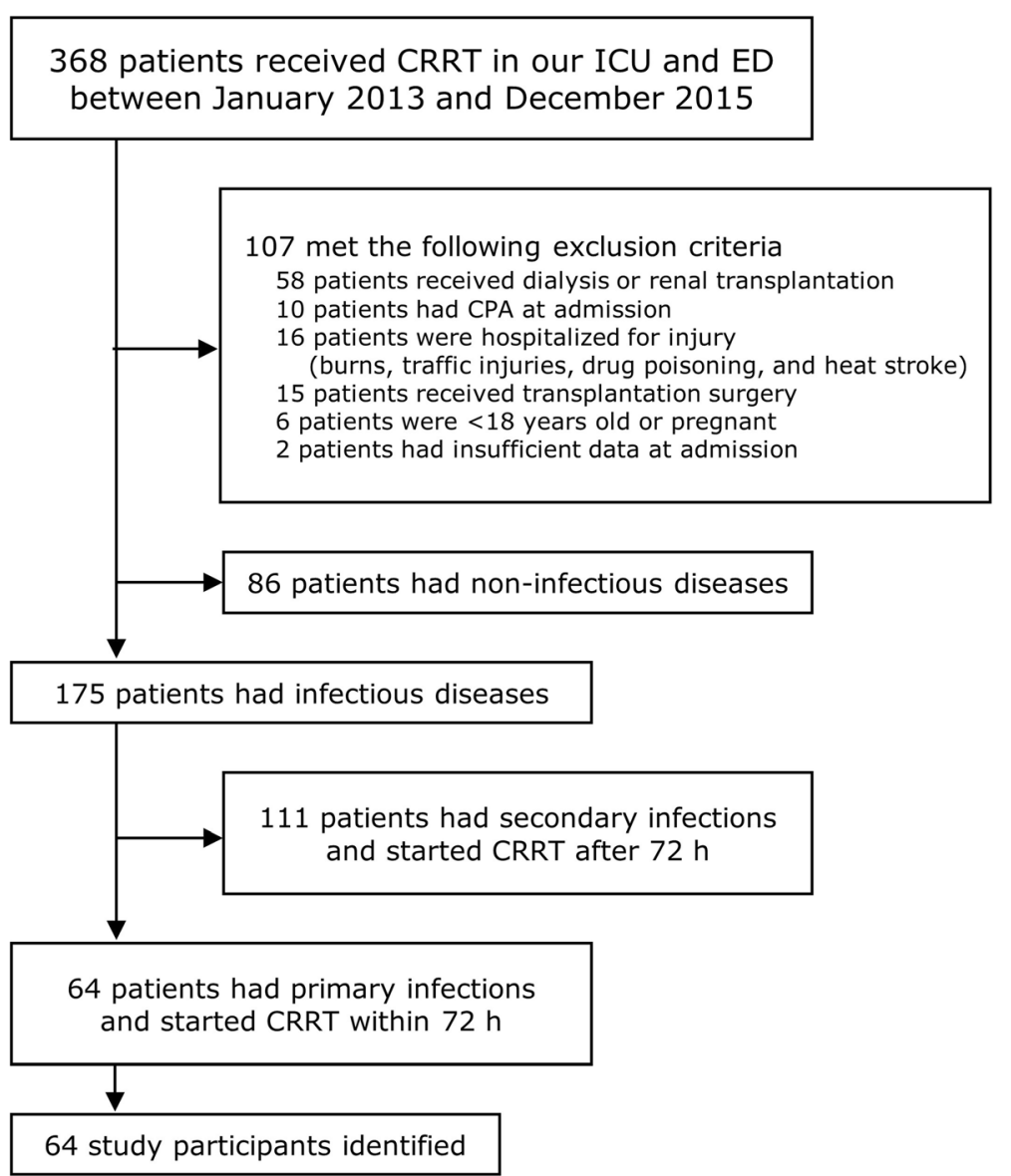

Fig. 1 Study recruitment flow chart. Abbreviations: CRRT continuous renal replacement therapy, ICU intensive care unit, ED emergency department, CPA cardiopulmonary arrest

more frequently used [23]. Sepsis was evaluated at admission and the start of CRRT. Using Sepsis-3 criteria, sepsis was diagnosed at admission in participant with a qSOFA score $\geq 2$, and at the start of CRRT in participants, a SOFA score $\geq 2$. Using Sepsis-1 criteria, sepsis was diagnosed in participants with any SIRS score $\geq 2$.

Septic shock was only evaluated at the start of CRRT, that is when reliable lactic acid levels were available. Using Sepsis-3 criteria, septic shock was diagnosed when vasopressor treatment was required to maintain a mean arterial pressure $\geq 65 \mathrm{mmHg}$ in the absence of hypovolemia and lactate levels were $>2 \mathrm{mmol} / \mathrm{L}$. Using Sepsis- 1 criteria, septic shock was diagnosed based on perfusion abnormalities and hypotension (a systolic blood pressure $<90 \mathrm{mmHg}$, a reduction in systolic blood pressure $\geq$ $40 \mathrm{mmHg}$ from baseline, or receiving vasopressor agents), despite adequate fluid resuscitation.

\section{Other definitions}

The severity of AKI was evaluated using serum creatinine levels, based on the Kidney Disease Improving Global Outcomes (KDIGO) classification [24]. Urine output and CRRT were not considered in this assessment. Baseline serum creatinine levels were defined as the level at the time of hospitalization, the most recent data from before admission, or the estimated serum creatinine level (based on the KDIGO guidelines) for patients with no information regarding prior kidney function.

The time of CRRT initiation was ascertained based on clinical status of each patient that included factors such as severe shock, increase in serum creatinine level, and decreased urine output. The attending physician determined the time of initiation and CRRT regimen.

All patients underwent central venous catheterization, and the dialysis membrane was changed every $24 \mathrm{~h}$, or earlier if clotting occurred.

Cultures collected within $48 \mathrm{~h}$ of admission or performed before hospitalization were used to identify causative pathogens. Patients were classified as having positive cultures if the same bacteria were detected in two cultures of blood or other specimens (e.g., sputum, urine, abdominal ascites, or pus). Any antimicrobial administered before admission was classed as pre-hospital antimicrobial therapy. 


\section{Outcome}

The primary outcome was 28-day mortality, and the secondary outcome was in-hospital mortality.

\section{Statistical analysis}

Data were reported as median (interquartile range) or number (\%). Statistical significance was set at $P<0.05$. Correlations were calculated using the non-parametric Spearman rank test. Fisher's exact test was used to assess the association of categorical variables with mortality. Univariate and multivariate logistic regression models were used to evaluate the relative risks of mortality, with results presented as odds ratios (OR) and 95\% confidence intervals. The factors identified as clinically related to the outcome on univariate analysis (based on $P$ value $\leq 0.20$ ) were included in the multivariate analysis. Receiver operating characteristic (ROC) curve analyses were performed to identify cutoff values, areas under the curve (AUC), sensitivities, and specificities. Survival was analyzed using Kaplan-Meier survival curves and the log-rank test. All analyses were performed using JMP Pro software version 12.2.0 (SAS Institute Inc.).

\section{Results}

\section{Participant characteristics}

The characteristics and laboratory data of the 64 participants $(70.3 \%$ male, median age 66.5 years) are shown in Table 1 . The median APACHE II score was 31 , the median SOFA score was 11, the median lactate level was $2.25 \mathrm{mmol} / \mathrm{L}$, and $51.6 \%$ of participants had received pre-hospital antimicrobial therapy.

The sources of infection and the culture results are shown in Table 2. The most common source of infection was pneumonia, followed by pancreatitis. Among the 27 participants $(42.2 \%)$ with positive cultures, the most common causal agents were Gram-positive bacteria, then Gram-negative bacteria. Most Gram-positive sepsis originated as a soft tissue infection or infective endocarditis, and most Gram-negative sepsis originated as a urinary tract infection. More than half of the participants received pre-hospital antimicrobial therapy, and no antimicrobial-resistant bacteria were detected within $48 \mathrm{~h}$ of admission.

Regarding the initial antimicrobial agents, Carbapenem was most commonly used in 40 cases (62.5\%), as the empiric antimicrobial therapy. About the number of initial antimicrobial agents, only one drug was used in 35 cases $(54.7 \%)$, and two drugs were used in 21 cases (32.8\%), respectively. In 8 cases $(12.5 \%)$, more than three drugs were used for initial treatment.

The details of the CRRT regimes are summarized in Table 3. Filters having high-adsorption capability, such as polymethylmethacrylate (PMMA) and AN69ST, were
Table 1 Participant characteristics

\begin{tabular}{|c|c|}
\hline Characteristic & All participants $(n=64)$ \\
\hline Age, years & $66.5(51-74.8)$ \\
\hline \multicolumn{2}{|l|}{ Sex } \\
\hline Male & $45(70.3 \%)$ \\
\hline Female & $19(29.7 \%)$ \\
\hline \multicolumn{2}{|l|}{ Comorbidities } \\
\hline Hypertension & $36(56.3 \%)$ \\
\hline Diabetes & $14(21.9 \%)$ \\
\hline Congestive heart failure & $11(17.2 \%)$ \\
\hline Liver disease & $15(23.4 \%)$ \\
\hline Smoking & $33(51.6 \%)$ \\
\hline Alcoholism & $8(12.5 \%)$ \\
\hline qSOFA & $1(1-2)$ \\
\hline SOFA & $11(8-13.8)$ \\
\hline APACHE ॥ & $31(24.3-36)$ \\
\hline Time from hospitalization to start of CRRT, min & $508(243-1395)$ \\
\hline Surgery before the start of CRRT* & $18(28.1 \%)$ \\
\hline Antimicrobial therapy before hospitalization & $33(51.6 \%)$ \\
\hline Positive blood cultures & $20(31.3 \%)$ \\
\hline Positive cultures $^{+}$ & $27(42.2 \%)$ \\
\hline \multicolumn{2}{|l|}{ Test results at the start of CRRT } \\
\hline Creatinine, mg/dL & $2.78(1.95-4.09)$ \\
\hline C-reactive protein, $\mathrm{mg} / \mathrm{dL}$ & $14.3(8.5-26.1)$ \\
\hline $\mathrm{pH}$ & $7.35(7.29-7.40)$ \\
\hline Lactate, mmol/L & $2.25(1.22-4.01)$ \\
\hline AKI at the start of CRRT & 47 (73.4\%) \\
\hline KDIGO stage 1 & 23 (35.9\%) \\
\hline KDIGO stage 2 & $8(12.5 \%)$ \\
\hline KDIGO stage 3 & $16(25.0 \%)$ \\
\hline Catecholamine index & $12(2.9-26.8)$ \\
\hline PMX-DHP combination & 20 (31.3\%) \\
\hline
\end{tabular}

Values are expressed as numbers (proportion) or median (interquartile range) Abbreviations: SOFA Sequential Organ Failure Assessment, APACHE Acute Physiology and Chronic Health Evaluation, CRRT continuous renal replacement therapy, AKI acute kidney injury, KDIGO Kidney Disease Improving Global Outcomes, PMX-DHP polymyxin B-immobilized fiber column direct hemoperfusion

*Emergency surgery to remove the source of infection as an unplanned operation

${ }^{\dagger}$ Positive cultures included blood cultures, cultures using other specimens, and pre-hospital findings

frequently used in 33 cases (51.6\%) and 11 cases (17.2\%), respectively.

\section{Diagnosing sepsis using Sepsis-1 and Sepsis-3 criteria, and those outcomes}

Figure 2 shows the diagnosis of sepsis using Sepsis-3 and Sepsis- 1 criteria. The Sepsis- 3 criteria identified 27 cases (42.2\%) at admission and 64 cases (100\%) at the 
Table 2 Sources of infection and culture results

\begin{tabular}{|c|c|}
\hline Source of infection & All participants $n=64$ ) \\
\hline Pneumonia & $13(20.3 \%)$ \\
\hline Pancreatitis & $9(14.1 \%)$ \\
\hline Hepatic and biliary tract infection & $8(12.5 \%)$ \\
\hline Urinary tract infection & $7(10.9 \%)$ \\
\hline Soft tissue infection & $7(10.9 \%)$ \\
\hline Gastrointestinal perforation & $6(9.4 \%)$ \\
\hline Infective endocarditis & $6(9.4 \%)$ \\
\hline Other & $8(12.5 \%)$ \\
\hline \multicolumn{2}{|l|}{ Culture results } \\
\hline Negative results & $37(57.8 \%)$ \\
\hline Positive results & $27(42.2 \%)$ \\
\hline Gram-positive organisms & $13(20.3 \%)$ \\
\hline Methicillin-susceptible Staphylococcus aureus & $5(7.8 \%)$ \\
\hline Enterococcus faecalis & $1(1.6 \%)$ \\
\hline Bacillus subtilis & $1(1.6 \%)$ \\
\hline Streptococcus constellatus & $2(3.1 \%)$ \\
\hline Streptococcus pyogenes & $1(1.6 \%)$ \\
\hline Streptococcus equisimilis & $1(1.6 \%)$ \\
\hline Streptococcus gallolyticus & $1(1.6 \%)$ \\
\hline Staphylococcus epidermidis & $1(1.6 \%)$ \\
\hline Gram-negative organisms & $12(18.8 \%)$ \\
\hline Escherichia coli & $5(7.8 \%)$ \\
\hline Klebsiella pneumoniae & $5(7.8 \%)$ \\
\hline Klebsiella oxytoca & $3(4.7 \%)$ \\
\hline Klebsiella planticola & $1(1.6 \%)$ \\
\hline Anaerobic organisms & $1(1.6 \%)$ \\
\hline Clostridium perfringens & $1(1.6 \%)$ \\
\hline Fungi & $1(1.6 \%)$ \\
\hline Candida glabrata & $1(1.6 \%)$ \\
\hline
\end{tabular}

Values are expressed as number (proportion)

start of CRRT (Fig. 2a). On the other hand, the Sepsis-1 criteria identified 53 cases $(82.8 \%)$ at admission, but only 44 cases $(68.8 \%)$ at the start of CRRT (Fig. 2b).

Among 64 participants, 20 (31.3\%) died within 28 days, and 28 (43.8\%) died in hospital. As shown in Table 4, the participants diagnosed with septic shock using the Sepsis-3 criteria had higher mortality rates than the participants without septic shock, both 28-day (46.0 vs $11.1 \%, P=0.003)$ and in-hospital mortality (62.2 vs $18.5 \%, P=0.0005$ ), respectively. On the other hand, Sepsis-1 criteria could not detect any significance for both 28 -day mortality ( 42.9 vs $25.6 \%, P=0.16$ ) and in-hospital mortality ( 52.4 vs $39.5 \%, P=0.33$ ). Sepsis-3 criteria were superior to Sepsis-1 in predicting survival.

The Kaplan-Meier survival curves are shown in Fig. 3. Participants diagnosed with septic shock using the Sepsis-
Table 3 Continuous renal replacement therapy regimens and treatment times

\begin{tabular}{ll}
\hline Regimen & All participants $(n=64)$ \\
\hline Filter & \\
PMMA & $33(51.6 \%)$ \\
AN69ST & $11(17.2 \%)$ \\
PS & $10(15.6 \%)$ \\
CTA & $5(7.8 \%)$ \\
PES & $5(7.8 \%)$ \\
CRRT dose, $\mathrm{mL} / \mathrm{kg} / \mathrm{h}$ & $17.0(13.6-19.0)$ \\
Duration of CRRT, days & $4.5(2-18)$ \\
Hospital stay, days & $40(7.3-59)$ \\
\hline
\end{tabular}

Values are expressed as number (proportion) or median (interquartile range) Abbreviations: CRRT continuous renal replacement therapy, PMMA polymethylmethacrylate, PS polysulfone, CTA cellulose triacetate, PES polyethersulfone

3 criteria had a significantly higher mortality rate than participants without septic shock (log-rank $P=0.0001$ ) (Fig. 3a), while no significant difference was observed using the Sepsis-1 criteria (Fig. 3b).

\section{Risk factors}

The univariate analyses revealed that both 28-day and in-hospital mortality were significantly associated with lactate levels $\geq 2 \mathrm{mmol} / \mathrm{L}$, SOFA scores $\geq 14$, and septic shock diagnosed using the Sepsis-3 criteria. Age > 65 years was only associated with in-hospital mortality (Tables 5 and 6). The multivariate analyses revealed that 28-day mortality was independently associated with lactate levels $\geq 2 \mathrm{mmol} / \mathrm{L}$, and pre-hospital antimicrobial therapy tended toward significance (Table 5), while in-hospital mortality was independently associated with age $>65$ years and lactate levels $\geq 2 \mathrm{mmol} / \mathrm{L}$ (Table 6).

As a continuous variable, lactate levels were strongly associated with in-hospital mortality. ROC analysis of in-hospital mortality revealed an optimal cutoff value for lactate levels of $1.8 \mathrm{mmol} / \mathrm{L}$ (AUC 0.777, sensitivity $85.7 \%$, specificity $58.3 \%$ ). In-hospital mortality was less strongly associated with SOFA score (AUC 0.67), APACHE II score (AUC 0.528), and age (AUC 0.637).

Among SOFA organ sub-scores, coagulation $(\mathrm{OR}=$ $1.55, P=0.046)$, hepatic function $(\mathrm{OR}=1.85, P=0.014)$, and central nervous system $(\mathrm{OR}=1.55, P=0.025)$ were significantly associated with 28 -day mortality, while respiratory, circulatory, and renal were not. Additionally, the hepatic function sub-score was significantly correlated with lactate levels (Spearman $\rho=0.35, P=0.005$ ). Regarding the source of infection, pneumonia which was the most common in this study was not associated with 28-day mortality, but it was significantly associated with in-hospital mortality $(\mathrm{OR}=3.79, P=0.046)$. 


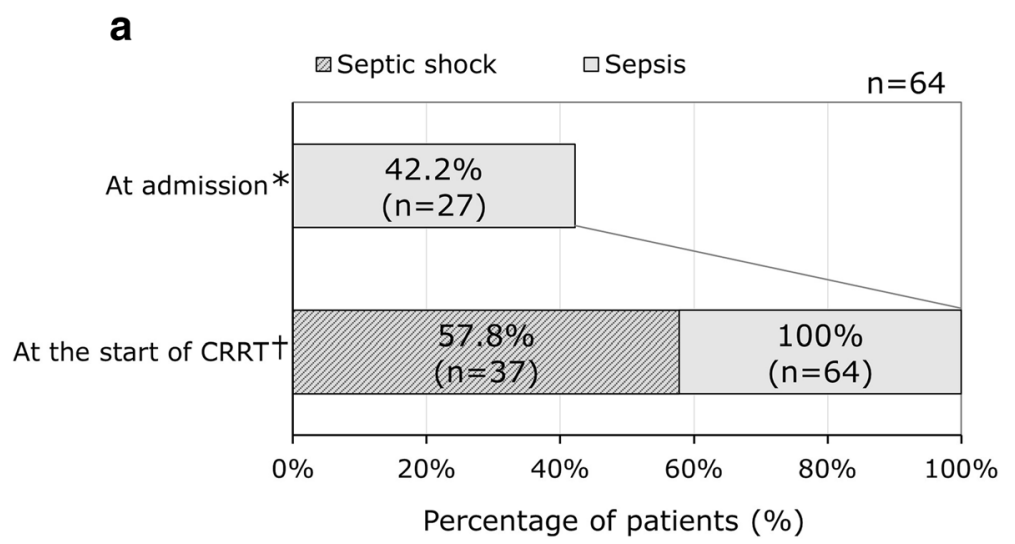

b

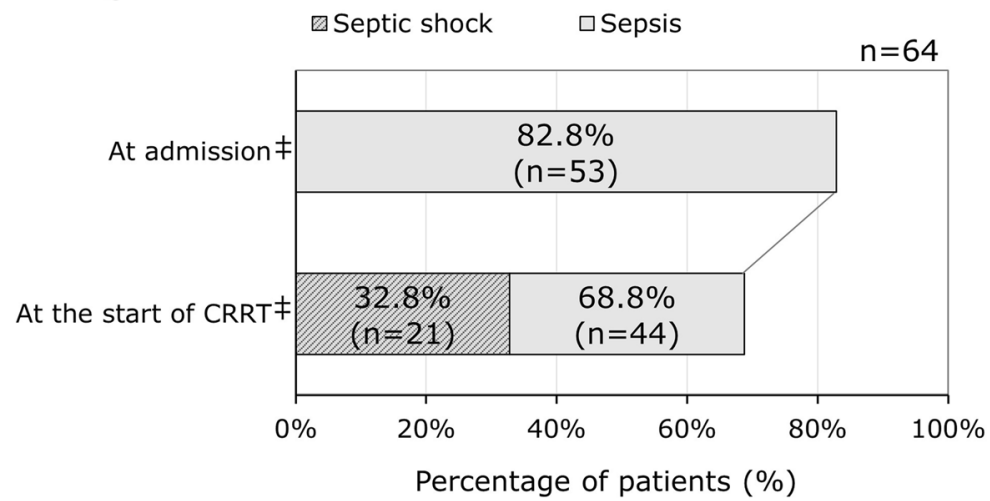

Fig. 2 Sepsis diagnoses using Sepsis-3 (a) and Sepsis-1 (b) criteria. Abbreviations: CRRT continuous renal replacement therapy, SOFA Sequential Organ Failure Assessment, SIRS systemic inflammatory response syndrome. ${ }^{*}$ Using the qSOFA score. ${ }^{+}$Using the SOFA score. ${ }^{*}$ Using the SIRS score

We did not detect any association between outcomes and regimen of CRRT, polymyxin B-immobilized fiber column direct hemoperfusion (PMX-DHP) combination, or general risk factors (e.g., AKI, serum creatinine levels, and comorbidities).

\section{Discussion}

The present study evaluated the usefulness of Sepsis-3 for predicting mortality among patients with primary infections who required CRRT. Mortality was associated with higher lactate levels, SOFA scores, and a septic shock diagnosis of Sepsis-3 criteria (lactate level $\geq$ $2 \mathrm{mmol} / \mathrm{L}$ ). Diagnosis with Sepsis-3 criteria is very accurate to discriminate septic shock from sepsis and is better for predicting survival than diagnosis with Sepsis-
1 criteria. In the present study, lactate levels were the most critical risk factor for the participants. Lactate levels are known to reflect the condition of critically ill patients, and their prognostic value had been identified in previous studies of patients with sepsis [25-27], and patients who required CRRT $[28,29]$. Our results support those findings.

According to Sepsis-3, sepsis is defined as a "life-threatening organ dysfunction caused by a dysregulated host response to infection" [19], and the participants of this study were in severe condition, requiring CRRT. As expected, using the Sepsis-3 criteria, all participants were diagnosed with sepsis based on their SOFA score at the start of CRRT. However, the qSOFA score at admission only identified $42.2 \%$ of these cases, and it was not

Table 4 Outcomes according to different criteria

\begin{tabular}{|c|c|c|c|c|c|}
\hline & \multirow{2}{*}{$\begin{array}{l}\text { All } \\
\text { participants }\end{array}$} & \multicolumn{2}{|l|}{ Sepsis-3 } & \multicolumn{2}{|l|}{ Sepsis-1 } \\
\hline & & Septic shock & Without septic shock & Septic shock & Without septic shock \\
\hline N & 64 & 37 & 27 & 21 & 43 \\
\hline 28-day mortality & $20(31.3 \%)$ & 17 (46.0\%) & $3(11.1 \%)$ & $9(42.9 \%)$ & $11(25.6 \%)$ \\
\hline In-hospital mortality & 28 (43.8\%) & $23(62.2 \%)$ & $5(18.5 \%)$ & $11(52.4 \%)$ & 17 (39.5\%) \\
\hline
\end{tabular}

Values are expressed as number (proportion) 


\section{a}

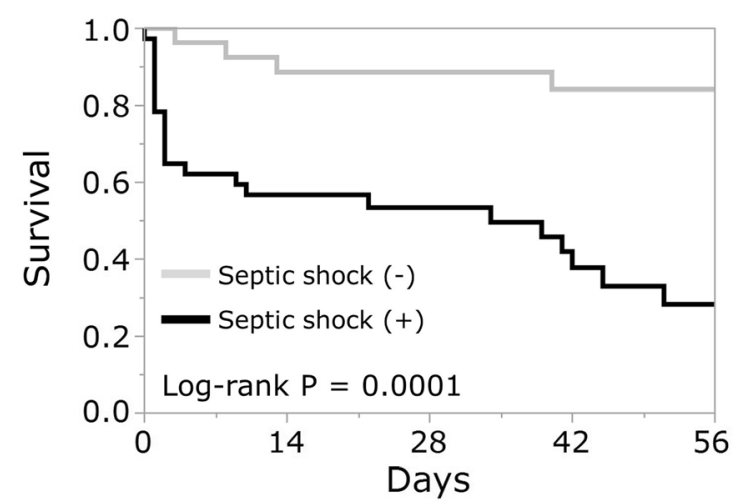

Number at risk

$\begin{array}{lllllc}\text { Septic shock }(-) & 27 & 24 & 23 & 19 & 14 \\ \text { Septic shock }(+) & 37 & 19 & 16 & 10 & 5\end{array}$

b

\section{Number at risk}

$$
\begin{aligned}
& \text { Septic shock (-) } \\
& \text { Septic shock (+) }
\end{aligned}
$$

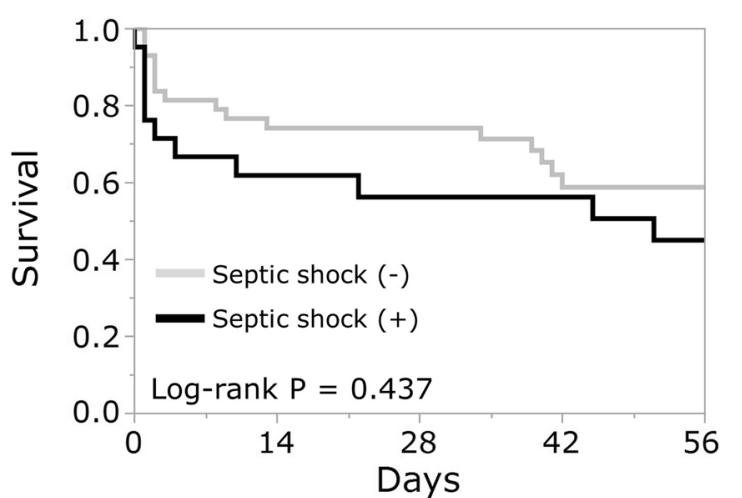


Table 5 Relative risk of 28-day mortality using univariate and multivariate logistic regression models

\begin{tabular}{|c|c|c|c|c|}
\hline \multirow[t]{3}{*}{ Variable } & \multicolumn{4}{|l|}{ 28-day mortality } \\
\hline & \multicolumn{2}{|l|}{ Univariate analysis } & \multicolumn{2}{|l|}{ Multivariate analysis } \\
\hline & Odds ratio $(95 \% \mathrm{Cl})$ & $P$ value & Odds ratio $(95 \% \mathrm{Cl})$ & $P$ value \\
\hline Age $\geq 65$ years & $2.80(0.91-8.63)$ & 0.1 & $2.61(0.77-9.80)$ & 0.12 \\
\hline Male sex & $0.98(0.31-3.11)$ & 1 & & \\
\hline Hypertension & $1.70(0.57-5.06)$ & 0.42 & & \\
\hline Diabetes & $0.30(0.06-1.47)$ & 0.19 & & \\
\hline Smoking & $2.68(0.89-8.04)$ & 0.1 & & \\
\hline Alcoholism & $0.28(0.03-2.43)$ & 0.41 & & \\
\hline Positive cultures & $0.65(0.22-1.93)$ & 0.59 & & \\
\hline Pre-hospital antimicrobial therapy & $2.22(0.75-6.65)$ & 0.18 & $3.10(0.92-11.7)$ & 0.068 \\
\hline Operation before starting CRRT & $0.79(0.24-2.64)$ & 0.77 & & \\
\hline PMX-DHP combination & $1.78(0.58-5.41)$ & 0.39 & & \\
\hline $\mathrm{AKI}$ at the start of CRRT & $0.55(0.17-1.74)$ & 0.37 & & \\
\hline Lactate $\geq 2 \mathrm{mmol} / \mathrm{L}$ & $6.80(1.74-26.6)$ & $0.003^{*}$ & $8.16(2.12-42.8)$ & $0.0015^{*}$ \\
\hline Mean blood pressure $\leq 65 \mathrm{mmHg}$ & $2.38(0.80-7.09)$ & 0.16 & & \\
\hline Use of catecholamine & $1.33(0.37-4.85)$ & 0.76 & & \\
\hline SOFA score $\geq 14$ & $6.33(1.85-21.6)$ & $0.0039^{*}$ & & \\
\hline APACHE $\|$ score $\geq 36$ & $3.18(1.01-10.0)$ & 0.07 & & \\
\hline Septic shock (Sepsis-3) & $6.80(1.74-26.6)$ & $0.003^{*}$ & & \\
\hline Septic shock (Sepsis-1) & $2.18(0.72-6.57)$ & 0.25 & & \\
\hline
\end{tabular}

Values are expressed as median (interquartile range)

The covariates in the multivariable-adjusted models were age $\geq 65$ years, pre-hospital antimicrobial therapy, and lactate $\geq 2 \mathrm{mmol} / \mathrm{L}$

Abbreviations: $C l$ confidence interval, CRRT continuous renal replacement therapy, PMX-DHP polymyxin B-immobilized fiber column direct hemoperfusion, AKI acute kidney injury, SOFA Sequential Organ Failure Assessment, APACHE Acute Physiology and Chronic Health Evaluation

${ }^{*} P<0.05$

this study, and they must have acidemia and/or high lactate levels because the medians (interquartile range) of $\mathrm{pH}$ and lactate were 7.35 (7.29-7.40) and $2.25(1.22-$ 4.01 ), respectively (Table 1 ). It is speculated that the initiation of CRRT was decided not only to supplement renal function but also to treat the critical multiple organ disorders associated with serious lactate retention. This is also suggested by the fact that the attending physicians tended to choose the hemofilter, which had the absorption characteristics.

This study also focused on the infection characteristics and the prognosis of the patients with communityacquired sepsis who required CRRT. Among various infectious diseases, pneumonia can easily cause hypoxia, and the hypoxia of multiple organs leads to serious lactate retention. This may be the reason why pneumonia was the most common source of infection in our study and the associated mortality was high among patients who required CRRT for community-acquired sepsis. Interestingly, positive culture results were not associated with mortality, while pre-hospital antimicrobial therapy had a clear trend as a risk factor for 28-day mortality. This result may reflect the fact that these patients started CRRT relatively late after the onset of their infection. Unfortunately, the present study was not able to accurately identify the timing of onset or pre-hospital course of the infection, so we cannot definitively comment on those relationships.

The present study had several limitations. First, because this was a single-center retrospective study, we only investigated a small number of patients with community-acquired sepsis who required CRRT and did not consider all patients with sepsis at our center. In order to simply compare Sepsis-1 and Sepsis-3 for diagnosis ability of sepsis, we need to extend the target population. Second, our findings may be biased because of the absence of standardized requirements for determining the time of CRRT initiation. Third, we did not measure cytokines or biomarkers relevant to sepsis cases, so we were unable to comment on underlying biology that might affect findings. Even if the sample size is small, we believe that the present study provides useful data for the following two reasons. Only a few studies have considered the Sepsis-3 criteria for diagnosing sepsis among patients who require CRRT, and the baseline data includes relatively uniform and detailed infectious characteristics because our cohort consists of a single-center community-acquired sepsis without in-hospital onset. 
Table 6 Relative risk of in-hospital mortality using univariate and multivariate logistic regression models

\begin{tabular}{|c|c|c|c|c|}
\hline \multirow[t]{3}{*}{ Variable } & \multicolumn{4}{|l|}{ In-hospital mortality } \\
\hline & \multicolumn{2}{|l|}{ Univariate analysis } & \multicolumn{2}{|l|}{ Multivariate analysis } \\
\hline & Odds ratio $(95 \% \mathrm{Cl})$ & $P$ value & Odds ratio $(95 \% \mathrm{Cl})$ & $P$ value \\
\hline Age $\geq 65$ years & $3.93(1.36-11.3)$ & $0.012^{*}$ & $3.96(1.22-14.3)$ & $0.021^{*}$ \\
\hline Male sex & $0.60(0.20-1.77)$ & 0.41 & & \\
\hline Hypertension & $1.38(0.51-3.77)$ & 0.61 & & \\
\hline Diabetes & $0.27(0.07-1.10)$ & 0.07 & & \\
\hline Smoking & $1.44(0.53-3.89)$ & 0.61 & & \\
\hline Alcoholism & $0.38(0.07-2.07)$ & 0.45 & & \\
\hline Positive cultures & $0.47(0.17-1.32)$ & 0.2 & & \\
\hline Pre-hospital antimicrobial therapy & $1.93(0.71-5.27)$ & 0.22 & $2.81(0.85-10.6)$ & 0.093 \\
\hline Operation before starting CRRT & $0.76(0.25-2.30)$ & 0.78 & & \\
\hline PMX-DHP combination & $1.44(0.50-4.18)$ & 0.59 & & \\
\hline $\mathrm{AKI}$ at the start of CRRT & $0.43(0.14-1.35)$ & 0.16 & & \\
\hline Lactate $\geq 2 \mathrm{mmol} / \mathrm{L}$ & $7.23(2.23-23.4)$ & $0.0008^{*}$ & $9.10(2.60-39.6)$ & $0.0003^{*}$ \\
\hline Mean blood pressure $\leq 65 \mathrm{mmHg}$ & $1.29(0.46-3.62)$ & 0.79 & & \\
\hline Use of catecholamine & $0.86(0.27-2.74)$ & 1 & & \\
\hline SOFA score $\geq 14$ & $6.00(1.67-21.6)$ & $0.0075^{*}$ & & \\
\hline APACHE $\|$ score $\geq 36$ & $1.94(0.65-5.85)$ & 0.27 & & \\
\hline Septic shock (Sepsis-3) & $7.23(2.23-23.4)$ & $0.0008^{*}$ & & \\
\hline Septic shock (Sepsis-1) & $1.68(0.59-4.82)$ & 0.42 & & \\
\hline
\end{tabular}

Values are expressed as median (interquartile range)

The covariates in the multivariable-adjusted models were age $\geq 65$ years, pre-hospital antimicrobial therapy, and lactate $\geq 2 \mathrm{mmol} / \mathrm{L}$

Abbreviations: $\mathrm{Cl}$ confidence interval, $C R R T$ continuous renal replacement therapy, $P M X-D H P$ polymyxin $\mathrm{B}$-immobilized fiber column direct hemoperfusion, $A K I$ acute kidney injury, SOFA Sequential Organ Failure Assessment, APACHE Acute Physiology and Chronic Health Evaluation $* P<0.05$

Considering the recent Japanese sepsis registry [31], which was successful in evaluating standard quality of care and mortality based on the international Surviving Sepsis Campaign guidelines, a similar prospective study of patients who required CRRT must be needed. Further research is required to validate our findings and to conduct a multicenter registry system considering the Sepsis-3 criteria for diagnosing sepsis among patients who required CRRT.

\section{Conclusions}

The experience at our center suggests that SOFA scores and lactate levels are strongly associated with mortality and that diagnosing septic shock with Sepsis-3 criteria is critical for predicting outcomes for patients who required CRRT for community-acquired sepsis. We concluded that in this population, Sepsis-3 criteria discriminated septic shock from sepsis very well, and it predicted outcomes, especially survival, more accurately than the previous criteria.

\section{Abbreviations}

AKI: Acute kidney injury; APACHE: Acute Physiology and Chronic Health Evaluation; AUC: Areas under the curve; Cl: Confidence interval; CPA: Cardiopulmonary arrest; CRRT: Continuous renal replacement therapy; CTA: Cellulose triacetate; ED: Emergency department;
ICU: Intensive care unit; KDIGO: The Kidney Disease Improving Global Outcomes; OR: Odds ratio; PES: Polyethersulfone; PMMA: Polymethylmethacrylate; PMX-DHP: Polymyxin B-immobilized fiber column direct hemoperfusion; PS: Polysulfone; qSOFA: Quick SOFA; ROC: Receiver operating characteristic; Sepsis-3: The Third Consensus Definitions of Sepsis and Septic Shock; SIRS: The systemic inflammatory response syndrome; SOFA: The Sequential Organ Failure Assessment

\section{Acknowledgements}

The authors thank all the nursing and medical staff (dialysis unit, ICU, and ED) for their patience and cooperation.

\section{Availability of data and materials}

All data generated or analyzed during this study are included in this published article and its supplementary information files.

\section{Authors' contributions}

MA collected and analyzed the data and wrote the manuscript. TY designed the study and helped to write the manuscript. MM, KW, EF, MN, HS, and SI helped to write the manuscript. All authors read and approved the final manuscript.

\section{Ethics approval and consent to participate}

The study protocol was approved by the Ethics Committee of the Tohoku University Graduate School of Medicine (2016-1-008).

\section{Consent for publication}

Not applicable.

Competing interests

The authors declare that they have no competing interests. 


\section{Publisher's Note}

Springer Nature remains neutral with regard to jurisdictional claims in published maps and institutional affiliations.

\section{Author details}

'Division of Nephrology, Endocrinology and Vascular Medicine, Tohoku University Graduate School of Medicine, 1-1 Seiryomachi, Aoba-ku, Sendai, Miyagi 980-8574, Japan. ${ }^{2}$ Division of Blood Purification, Tohoku University Hospital, Sendai, Japan. ${ }^{3}$ Research Division of Chronic Kidney Disease and Dialysis Treatment, Tohoku University Hospital, Sendai, Japan.

Received: 1 May 2018 Accepted: 7 September 2018 Published online: 21 September 2018

\section{References}

1. Uchino S, Kellum JA, Bellomo R, Doig GS, Morimatsu H, Morgera S, Schetz M, Tan I, Bouman C, Macedo E, et al. Acute renal failure in critically ill patients: a multinational, multicenter study. JAMA. 2005;294:813-8.

2. Bagshaw SM, George C, Bellomo R. Early acute kidney injury and sepsis: a multicentre evaluation. Crit Care. 2008;12:R47.

3. Bagshaw SM, Uchino S, Bellomo R, Morimatsu H, Morgera S, Schetz M, Tan I, Bouman C, Macedo E, Gibney N, et al. Septic acute kidney injury in critically ill patients: clinical characteristics and outcomes. Clin J Am Soc Nephrol. 2007;2:431-9.

4. Nagata I, Uchino S, Tokuhira N, Ohnuma T, Namba Y, Katayama S, Kawarazaki $\mathrm{H}$, Toki N, Takeda K, Yasuda H, et al. Sepsis may not be a risk factor for mortality in patients with acute kidney injury treated with continuous renal replacement therapy. J Crit Care. 2015;30:998-1002.

5. Neveu $H$, Kleinknecht D, Brivet F, Loirat P, Landais P. Prognostic factors in acute renal failure due to sepsis. Results of a prospective multicentre study. The French Study Group on Acute Renal Failure. Nephrol Dial Transplant. 1996;11:293-9.

6. Schetz M. Non-renal indications for continuous renal replacement therapy. Kidney Int Suppl. 1999;56:S88-94.

7. Dellinger RP, Levy MM, Rhodes A, Annane D, Gerlach H, Opal SM, Sevransky JE, Sprung CL, Douglas IS, Jaeschke R, et al. Surviving sepsis campaign: international guidelines for management of severe sepsis and septic shock: 2012. Crit Care Med. 2013;41:580-637.

8. Ferrer R, Artigas A, Suarez D, Palencia E, Levy MM, Arenzana A, Perez XL, Sirvent JM, Edusepsis Study G. Effectiveness of treatments for severe sepsis: a prospective, multicenter, observational study. Am J Respir Crit Care Med. 2009;180:861-6.

9. Gaieski DF, Mikkelsen ME, Band RA, Pines JM, Massone R, Furia FF, Shofer FS, Goyal M. Impact of time to antibiotics on survival in patients with severe sepsis or septic shock in whom early goal-directed therapy was initiated in the emergency department. Crit Care Med. 2010;38:1045-53.

10. Karvellas CJ, Farhat MR, Sajjad I, Mogensen SS, Leung AA, Wald R, Bagshaw SM. A comparison of early versus late initiation of renal replacement therapy in critically ill patients with acute kidney injury: a systematic review and metaanalysis. Crit Care. 2011;15:R72

11. Zarbock A, Kellum JA, Schmidt C, Van Aken H, Wempe C, Pavenstadt H, Boanta A, Gerss J, Meersch M. Effect of early vs delayed initiation of renal replacement therapy on mortality in critically ill patients with acute kidney injury: the ELAIN randomized clinical trial. JAMA. 2016;315:2190-9.

12. Gaudry S, Hajage D, Schortgen F, Martin-Lefevre L, Pons B, Boulet E, Boyer A, Chevrel G, Lerolle N, Carpentier D, et al. Initiation strategies for renalreplacement therapy in the intensive care unit. N Engl J Med. 2016;375:122-33.

13. Heering P, Morgera S, Schmitz FJ, Schmitz G, Willers R, Schultheiss HP, Strauer BE, Grabensee B. Cytokine removal and cardiovascular hemodynamics in septic patients with continuous venovenous hemofiltration. Intensive Care Med. 1997;23:288-96.

14. Hoffmann JN, Hartl WH, Deppisch R, Faist E, Jochum M, Inthorn D. Effect of hemofiltration on hemodynamics and systemic concentrations of anaphylatoxins and cytokines in human sepsis. Intensive Care Med. 1996;22:1360-7.

15. Honore PM, Joannes-Boyau O, Boer W, Collin V. High-volume hemofiltration in sepsis and SIRS: current concepts and future prospects. Blood Purif. 2009;28:1-11.

16. Perez-Fernandez X, Sabater-Riera J, Sileanu FE, Vazquez-Reveron J, BallusNoguera J, Cardenas-Campos P, Betbese-Roig A, Kellum JA. Clinical variables associated with poor outcome from sepsis-associated acute kidney injury and the relationship with timing of initiation of renal replacement therapy. $J$ Crit Care. 2017;40:154-60.
17. Bone RC, Balk RA, Cerra FB, Dellinger RP, Fein AM, Knaus WA, Schein RM, Sibbald WJ. Definitions for sepsis and organ failure and guidelines for the use of innovative therapies in sepsis. The ACCP/SCCM Consensus Conference Committee. American College of Chest Physicians/Society of Critical Care Medicine. Chest. 1992;101:1644-55.

18. Levy MM, Fink MP, Marshall JC, Abraham E, Angus D, Cook D, Cohen J, Opal SM, Vincent JL, Ramsay G. International Sepsis Definitions C: 2001 SCCM/ ESICM/ACCP/ATS/SIS International Sepsis Definitions Conference. Intensive Care Med. 2003;29:530-8.

19. Singer M, Deutschman CS, Seymour CW, Shankar-Hari M, Annane D, Bauer M, Bellomo R, Bernard GR, Chiche JD, Coopersmith CM, et al. The Third International Consensus Definitions for Sepsis and Septic Shock (Sepsis-3). JAMA. 2016;315:801-10.

20. Besen BA, Romano TG, Nassar AP Jr, Taniguchi LU, Azevedo LC, Mendes PV, Zampieri FG, Park M. Sepsis-3 definitions predict ICU mortality in a lowmiddle-income country. Ann Intensive Care. 2016;6:107.

21. Knaus WA, Draper EA, Wagner DP, Zimmerman JE. APACHE II: a severity of disease classification system. Crit Care Med. 1985;13:818-29.

22. Vincent JL, Moreno R, Takala J, Willatts S, De Mendonca A, Bruining H, Reinhart CK, Suter PM, Thijs LG. The SOFA (Sepsis-related organ failure assessment) score to describe organ dysfunction/failure. On behalf of the Working Group on Sepsis-Related Problems of the European Society of Intensive Care Medicine. Intensive Care Med. 1996;22:707-10.

23. Zhao H, Heard SO, Mullen MT, Crawford S, Goldberg RJ, Frendl G, Lilly CM An evaluation of the diagnostic accuracy of the 1991 American College of Chest Physicians/Society of Critical Care Medicine and the 2001 Society of Critical Care Medicine/European Society of Intensive Care Medicine/ American College of Chest Physicians/American Thoracic Society/Surgical Infection Society sepsis definition. Crit Care Med. 2012;40:1700-6.

24. KDIGO. Clinical practice guideline for acute kidney injury. Kidney Int Suppl. 2012;2:1-138.

25. Jansen TC, van Bommel J, Schoonderbeek FJ, Sleeswijk Visser SJ, van der Klooster JM, Lima AP, Willemsen SP, Bakker J, group LS. Early lactate-guided therapy in intensive care unit patients: a multicenter, open-label, randomized controlled trial. Am J Respir Crit Care Med. 2010;182:752-61.

26. Mikkelsen ME, Miltiades AN, Gaieski DF, Goyal M, Fuchs BD, Shah CV, Bellamy SL, Christie JD. Serum lactate is associated with mortality in severe sepsis independent of organ failure and shock. Crit Care Med. 2009;37: 1670-7.

27. Shankar-Hari M, Phillips GS, Levy ML, Seymour CW, Liu VX, Deutschman CS, Angus DC, Rubenfeld GD, Singer M, Sepsis Definitions Task F. Developing a new definition and assessing new clinical criteria for septic shock: for the Third International Consensus Definitions for Sepsis and Septic Shock (Sepsis-3). JAMA. 2016;315:775-87.

28. Kawarazaki H, Uchino S, Tokuhira N, Ohnuma T, Namba Y, Katayama S, Toki $\mathrm{N}$, Takeda K, Yasuda H, Izawa J, et al. Who may not benefit from continuous renal replacement therapy in acute kidney injury? Hemodial Int. 2013;17: 624-32.

29. Passos RD, Ramos JG, Gobatto A, Mendonca EJ, Miranda EA, Dutra FR, Coelho MF, Pedroza AC, Batista PB, Dutra MM. Lactate clearance is associated with mortality in septic patients with acute kidney injury requiring continuous renal replacement therapy: a cohort study. Medicine (Baltimore). 2016;95:e5112.

30. Smith OM, Wald R, Adhikari NK, Pope K, Weir MA, Bagshaw SM. Standard versus accelerated initiation of renal replacement therapy in acute kidney injury (STARRT-AKI): study protocol for a randomized controlled trial. Trials. 2013;14:320.

31. Fujishima S, Gando S, Saitoh D, Mayumi T, Kushimoto S, Shiraishi S, Ogura $\mathrm{H}$, Takuma K, Kotani J, Ikeda H, et al. A multicenter, prospective evaluation of quality of care and mortality in Japan based on the Surviving Sepsis Campaign guidelines. J Infect Chemother. 2014;20:115-20. 